\title{
Estimating the sensitivity of the expanded OVRO-LWA array to cosmic-ray primary composition
}

\author{
W. R. Carvalho jr.* ${ }^{a b}$, A. Romero-Wolf ${ }^{c}$, K. Belov ${ }^{c}$, L. D’Addario ${ }^{a}$, M. Anderson ${ }^{a}$, M.

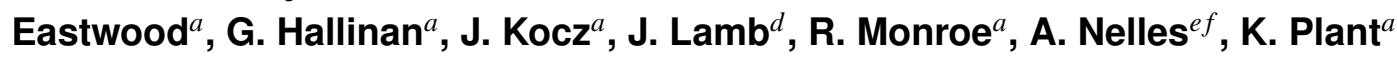 \\ ${ }^{a}$ Department of Astronomy - California Institute of Technology, Pasadena CA, USA \\ ${ }^{b}$ Physics Institute, University of São Paulo, São Paulo, Brazil \\ ${ }^{c}$ Jet Propulsion Laboratory, California Institute of Technology, Pasadena CA, USA \\ ${ }^{d}$ California Institute of Technology, Owens Valley Radio Observatory, Big Pine CA, USA \\ ${ }^{e}$ ECAP, FAU Erlangen, Germany \\ ${ }^{f}$ DESY, Zeuthen, Germany
}

E-mail: carvajregmail.com

\begin{abstract}
In this study we investigate the capability of the planned expansion of the Owens Valley Long Wavelength Array (OVRO-LWA) to perform radio based composition studies of the cosmic-ray flux between $50 \mathrm{PeV}$ and $1 \mathrm{EeV}$. We use ZHAIRES simulations in conjunction with a method similar to the one developed by the LOFAR experiment, but that only uses radio data between 30 and $80 \mathrm{MHz}$, to reconstruct the depth of shower maximum $X_{\max }$. We found that the lower antenna densities away from the central core of the array lead to a decrease in the effective area for quality $X_{\max }$ reconstructions of low zenith angle events, and that the asymmetry of the array layout creates the need for quality cuts that depend on arrival direction. We also investigate the dependence of $X_{\max }$ uncertainties on shower energy and arrival direction and show that at 500 $\mathrm{PeV}$ it is possible to obtain $X_{\max }$ uncertainties lower than $20 \mathrm{~g} / \mathrm{cm}^{2}$ for the majority of arrival directions by using a set of simple cuts. For energies below $10^{17} \mathrm{eV}$, alternative reconstruction methods, such as the use of hierarchical beamforming to increase SNR or the use of arrival time measurements on the ground may be needed to maintain $X_{\max }$ resolution at a desirable level.
\end{abstract}

36th International Cosmic Ray Conference -ICRC2019-

July 24th - August 1st, 2019

Madison, WI, U.S.A.

${ }^{*}$ Speaker. 


\section{Introduction}

OVRO-LWA currently features 288 crossed broadband dipole antennas. Most are deployed in a very dense $200 \mathrm{~m}$ diameter center core, but 32 expansion antennas extend beyond the core to create baselines of up to $1.5 \mathrm{~km}$. A recently developed radio only trigger approach based on radio frequency interference (RFI) rejection has shown to be effective in detecting cosmic-ray events in its somewhat noisy environment (see [1] and the PoS(ICRC2019)405 proceedings of Romero-Wolf et al.). The array is now undergoing an expansion that will increase the number of antennas to 352 , increasing the density of antennas beyond the core and the maximum baseline to $2.6 \mathrm{~km}$. A new signal processing infrastructure consisting of more powerful electronics, such as new Field-programmable gate arrays (FPGAs), will allow the continuous detection of cosmic rays commensally with the other science goals of the observatory (see the PoS(ICRC2019)391 proceedings of K. Plant et al.). In this work we use simulations to estimate the expanded OVROLWA sensitivity to cosmic-ray composition, based on radio reconstructions of the depth of shower maximum $X_{\max }$.

Some composition measurements in the second knee region of the cosmic ray flux, between $\sim 30 \mathrm{PeV}$ and $1 \mathrm{EeV}$, suggest a transition from a heavy to a light composition at around $100 \mathrm{PeV}$ (see [2] and references therein). One interpretation is that this marks the transition between galactic and extra-galactic fluxes: As galactic sources become unable to accelerate even heavy nuclei above $100 \mathrm{PeV}$, the flux becomes dominated by the low mass component of the extra-galactic flux [2]. A very precise measurement of cosmic-ray composition and anisotropy in this region would shed light into this interpretation. Also, a search for small-angle clustering of cosmic-ray events consistent with neutron primaries could be able to pinpoint galactic cosmic-ray sources. Due to the effect of time dilation on neutron decay, the energy of such events could be used to constrain the distance to the source, making it possible to map galactic cosmic-ray sources using neutrons [3]. All this points to the necessity of very precise composition measurements at these lower energies.

\section{Radio emission and detector simulations}

In this work we used ZHAIRES [4], a first-principles microscopic Monte-Carlo simulation of the radio emission of air showers, to perform simulations for several arrival directions by sweeping zenith angles between $0^{\circ}$ and $60^{\circ}$ in $10^{\circ}$ steps for the whole azimuth range in steps of $45^{\circ}$. For each geometry we performed 50 proton- and 50 iron-induced simulations using the following parameters: Shower energy of $5 \times 10^{17} \mathrm{eV}$, thinning level of $10^{-5}$, thinning weight factor 0.06 , time bin size of $0.3 \mathrm{~ns}$ and a $e^{ \pm}$and $\gamma$ cuts of $80 \mathrm{keV}$ at the OVRO-LWA site, with ground altitude of $1200 \mathrm{~m}$ and a geomagnetic field $|\vec{B}|=48.5 \mu T$ with an inclination of $61.6^{\circ}$. We also used the superposition model described in [5] to estimate the amplitude and polarization of the peak net electric field at any position on the ground, which is needed by the minimization procedure described in Section 3.

We generated input events by convoluting the peak electric fields obtained from the simulations with the detector response to galactic noise. To relate the measured voltage at the antenna front end to the incident electric field we use the effective height of the antenna $h_{\text {eff }}(\lambda, \theta)$ (see [1]). Instead of using the full wavelength dependence of the effective height, we approximate it by the average value over the $30-80 \mathrm{MHz}$ band, obtaining $\left\langle h_{\text {eff }}\right\rangle \simeq 0.47$. We also assume that the antenna beam pattern is known well enough, so that the uncertainty in the reconstructed electric field is dominated 
by galactic noise. This means that we neglect any errors in directivity and arrival direction and assume that the effect of the directivity term when generating an input event cancels out with the same effect when reconstructing the electric field from the voltage trace. We used the noise voltage spectrum $|V|_{\text {noise }}^{2}$ and the root-mean-square noise $V_{\mathrm{RMS}}$ at the front end of the detector, as described in [1], to obtain the value $V_{\mathrm{RMS}} \simeq 11.5 \mu \mathrm{V}$. The noise contribution $E_{R M S}$ to the peak electric field is then given by $E_{R M S}=V_{R M S} /\left\langle h_{\mathrm{eff}}\right\rangle \simeq 25 \mu \mathrm{V} / \mathrm{m}$ per component of the electric field.

The spatial layout of the antennas in the OVRO-LWA-352 expansion can be seen in the top left panel of Fig. 1. For each input event we sample the core position equally distributed in the area of the array and use a ZHAIRES simulation convoluted with the expected noise in conjunction with the superposition model to estimate the peak electric field in the N-S and E-W polarizations at each antenna position w.r.t. the sampled core, neglecting any uncertainties in arrival direction.

\section{Event Reconstruction}

To reconstruct $X_{\max }$, we use a method based on comparisons between measured and simulated electric fields at multiple antennas, very similar to that developed by LOFAR [6], but that only uses radio data. Multiple proton and iron simulations with the same energy and arrival direction as the detected event are compared to it. To quantify the differences between data and simulation we use a variable called $\Sigma_{s}$, defined as the quadratic sum of the differences between the measured and simulated peak power over all antennas with signal:

$$
\Sigma_{s}\left(f_{s}, \vec{r}_{\text {core }}\right)=\sum_{\text {antennas }}\left[\left|\vec{E}_{\text {data }}\right|^{2}-f_{s}^{2} \cdot\left|\vec{E}_{\mathrm{MC}}\right|^{2}\left(x-x_{\text {core }}, y-y_{\text {core }}\right)\right]^{2},
$$

where $f_{s}$ is an energy scaling factor, $\left|\vec{E}_{\text {data }}\right|$ and $\left|\vec{E}_{\mathrm{MC}}\right|$ are the measured and simulated peak electric field at each antenna, respectively. The peak electric field is defined as the peak of the Hilbert envelope of the time-domain signal. The core position is $\vec{r}_{\text {core }}=\left(x_{\text {core }}, y_{\text {core }}\right)$ and the antenna position is $(x, y)$.

This method needs a prior estimate of the energy and core position of the event, both subject to uncertainties (see section 3.1). To account for these uncertainties, we vary the position of the core and the energy scaling factor during minimization. This leads to multiple values of $\Sigma_{s}\left(f_{s}, \vec{r}_{\text {core }}\right)$ for a single simulation, but only the minimum value, denoted simply as $\Sigma$, is used. It corresponds to the values of $f_{s}$ and $\left(x_{\text {core }}, y_{\text {core }}\right)$ for which the simulation best represents the detected event. Finally, $\Sigma$ is plotted as a function of the known value of $X_{\max }$ for each individual simulation and a 4th degree polynomial fit is performed. The position of the minimum of the fit is taken as the reconstructed $X_{\max }$ for the event. Better estimates for the energy and core position of the event are byproducts of this type of $X_{\max }$ reconstruction, improving the initial estimates described in Section 3.1. The minimized value of $\vec{r}_{\text {core }}$ is taken as the reconstructed core position, while the minimized value of $f_{s}$ is used to obtain the final reconstructed energy $E_{\text {rec }}=f_{s} E_{\text {sim }}$, where $E_{\text {sim }}$ is the energy of the simulation. In the top right and bottom panels of Fig. 1 we show the reconstruction of an event (see caption for details).

\subsection{Initial core position and energy estimates}

To perform the full $X_{\max }$ reconstruction, estimates of the energy and core position are needed to produce the simulation set and constrain the region for the final core minimization. Given an event 

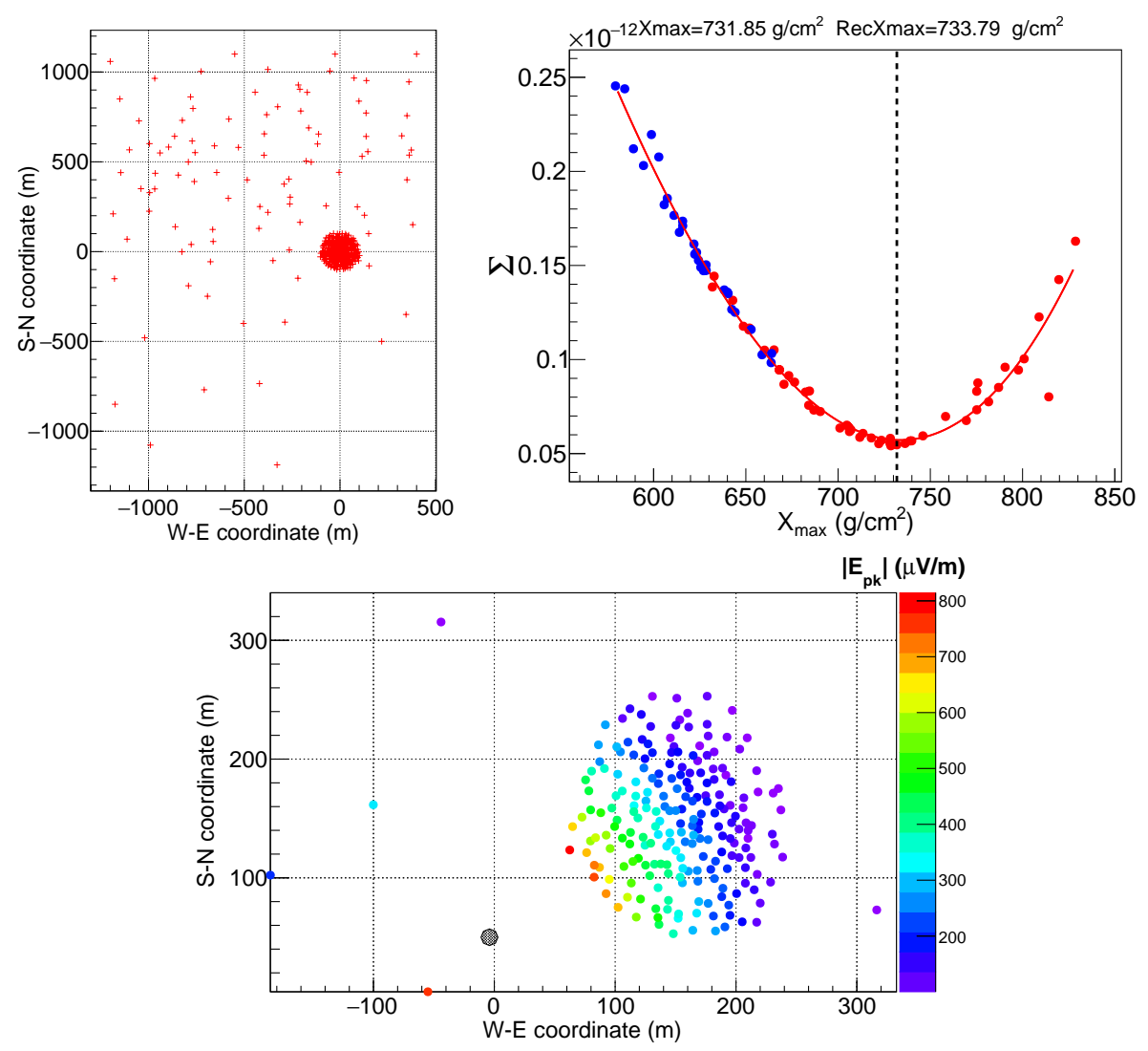

Figure 1: Top left: Antenna position map for the OVRO-LWA-352 expansion. Top right: Reconstruction example of a $500 \mathrm{PeV}$ proton shower with $\theta=30^{\circ}$ coming from the North, showing $\Sigma$ as a function of $X_{\max }$. Each point in the plot corresponds to one of the 50 proton (red) or 30 iron (blue) ZHAIRES simulations used to reconstruct the event. The reconstructed value of $X_{\max }$ is the position of the minimum of the polynomial fit and the vertical dashed line represents the known $X_{\max }$ of the input event. Bottom: Map of the accepted antennas for this same event. The color scale is the amplitude of the horizontal component of the peak electric field and the grey circle is the minimized core position w.r.t. the initial estimate at $(0,0)$.

and its arrival direction, we first calculate the baricenter of the signal, i.e. the average coordinate of the accepted antennas weighted by their signal. We then compare the detected event to a single proton simulation with a fixed energy $E_{\text {sim }}=5 \times 10^{17} \mathrm{eV}$ by minimizing $\Sigma_{E}$ (Eq. 3.2), a very similar variable to $\Sigma_{s}$ (Eq. 3.1), but that uses the peak electric field instead of peak power. Here we constrain the core minimization in a large area around the previously calculated baricenter. The minimized values of $f_{s}$ and $\vec{r}_{\text {core }}$ are then taken as the first estimates of the event energy $\left(E=f_{s} E_{\text {sim }}\right)$ and core position, respectively. Error distributions of these estimates, based on simulations of $30^{\circ}$ showers, are shown in Fig. 2. One can see that the energy resolution is very good, around 20\%, even when minimizing iron input events against proton-induced simulations. Our results for the core error, defined as the distance between the known and the reconstructed core positions, show a resolution around $20 \mathrm{~m}$, even when mismatching the input and simulation compositions. All input events were created as described in section 2, so we assume a perfect calibration of the detector and disregard arrival direction uncertainties. 


$$
\Sigma_{E}\left(f_{s}, \vec{r}_{\text {core }}\right)=\sum_{\text {antennas }}\left[\left|\vec{E}_{\text {data }}\right|-f_{s} \cdot\left|\vec{E}_{\mathrm{MC}}\right|\left(x-x_{\text {core }}, y-y_{\text {core }}\right)\right]^{2}
$$
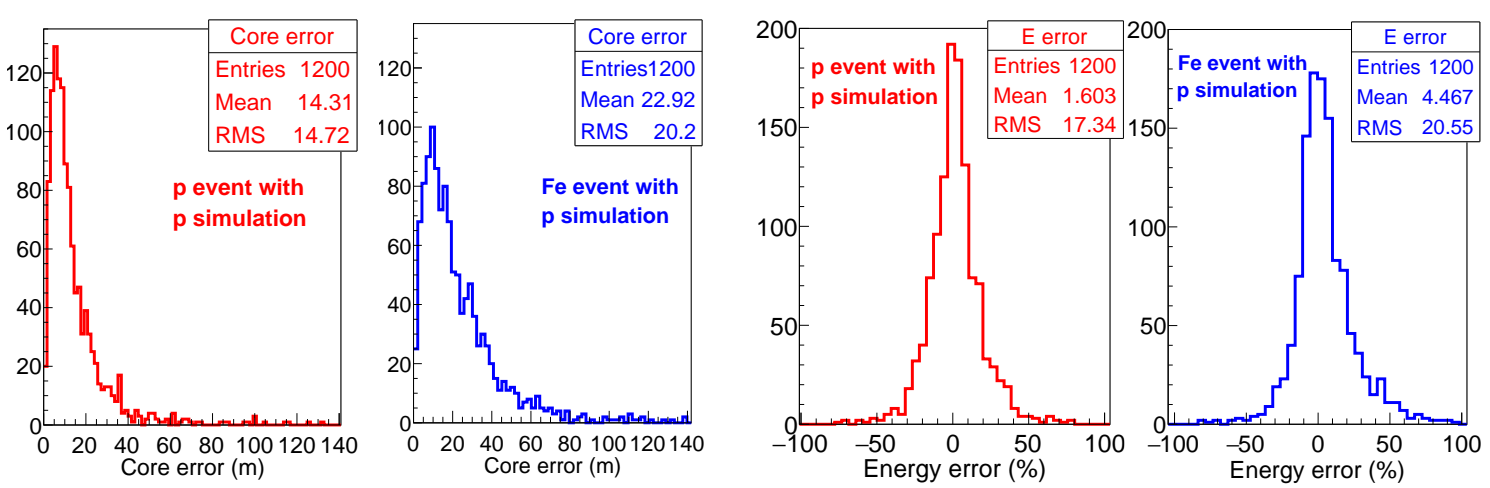

Figure 2: Error distributions for the first estimates of core position and energy, based on 1200 reconstructions of proton and 1200 iron events with $\theta=30^{\circ}$. Left: Core position error distributions for proton (red) and iron (blue) events. Right: Energy error distributions for proton (red) and iron (blue) events. The shower simulation used for the reconstruction is always proton-induced.

\section{Expected Performance}

\subsection{Dependence on arrival direction}

The radio emission of air showers is dominated by the geomagnetic mechanism, which roughly scales with $\sin \alpha$, where $\alpha$ is the angle between the shower axis and the geomagnetic field [7]. On the top left panel of Fig. 3 we show $\sin \alpha$ as a function of azimuth angle $\phi$ and for several zenith angles $\theta$ at the OVRO-LWA site. In addition, since the OVRO-LWA-352 array is not symmetric, the number and position of triggered antennas strongly depend not only on signal strength, but also on the geometrical shape and orientation of the footprint. Also, as shown in [8], the variation of the radio footprint with the $X_{\max }$ of the shower diminishes as the shower zenith angle increases, disfavoring $X_{\max }$ reconstructions of more inclined events. All this points to a strong dependence of the $X_{\max }$ uncertainty on shower arrival direction. To study this we have performed simulations of the $X_{\max }$ reconstruction for several arrival directions.

The OVRO-LWA-352 array has a very dense core of antennas, but a much more sparse distribution away from the center core (see top left panel of Fig. 1). We found that this leads to two different regimes of detection. Lower zenith angles $\left(\theta \lesssim 30^{\circ}\right)$ produce small footprints that can only be properly sampled by the dense central core of the array, leading to increased uncertainties as the shower core moves away from the center of the array. On the other hand, larger zenith angle showers $\left(\theta \gtrsim 40^{\circ}\right)$ produce larger radio footprints that can be properly sampled even by the less dense parts of the array. To account for this we created a set of basic quality cuts that require a minimum number of triggered antennas and vary with zenith angle: For events with zenith angles $\theta \leq 30^{\circ}$ we enforced a minimum of 70 triggered antennas, for the transition region at $\theta=40^{\circ}$ a minimum of 30 antennas, while for $\theta>40^{\circ}$ the threshold is lowered to only 10 antennas.

On the middle left panel of Fig. 3 we show the uncertainty in $X_{\max }\left(\sigma_{X_{\max }}\right)$ as a function of azimuth angle for several zenith angles. Each point represents 250 events that pass the basic cuts, 
created as described in section 2 and then reconstructed as described in Section 3. One can see that, as expected, showers coming from the South $(\phi=180)$, tend to have larger uncertainties due to the lower SNR brought by the $\sin \alpha$ dependence of the electric field. Also, smaller zenith angles tend to produce better $X_{\max }$ reconstructions, if compared to more inclined events. Although the basic cuts on the number of antennas are enough to reject extremely bad reconstructions, harsher cuts that also depend on arrival direction can be applied to obtain a subset of events with even lower $X_{\max }$ uncertainties. For lower zenith angles these harsher cuts take the form of an increased minimum number of antennas ${ }^{1}$. For more inclined events, further cuts on the number of antennas do not work well, and the harsher cuts are based on spatial cuts that mainly reject events with the shower core close to the borders of the array ${ }^{2}$. These harsher cuts further remove $\sim 25-60 \%$ of the events that passed the initial basic cuts, depending on the arrival direction. The middle right panel of Fig. 3 shows the results of applying the stronger cuts to the same simulation sets. One can see that applying the stronger cuts leads to $\sigma_{X_{\max }}<20 \mathrm{~g} / \mathrm{cm}^{2}$ for the majority of geometries, with some low zenith angle geometries reaching values below $15 \mathrm{~g} / \mathrm{cm}^{2}$.

The core position reconstruction of these events also showed a very good resolution, with typical uncertainties $\sim 5 \mathrm{~m}$ for low zenith angles, increasing to an average of only $\sim 17 \mathrm{~m}$ at $60^{\circ}$, even when only the basic cuts are applied. This explains why the impact of a core position uncertainty in $\sigma_{X_{\max }}$ was very modest. Comparisons between reconstructions with and without a folded core uncertainty showed only a small difference in $\sigma_{X_{\max }}$, typically lower than $\sim 2 \mathrm{~g} / \mathrm{cm}^{2}$.

By integrating our results over all arrival directions, we obtained the expected event rate at 500 $\mathrm{PeV}$, as can be seen in the top right panel of Fig. 3. The lower event rates of less inclined shower are mainly due to the lower effective area of the detector for these events, since they have to land near the central core of the array. We expect much larger event rates at lower energies.

\subsection{Energy dependence}

Since signal strength increases with energy, it also affects $X_{\max }$ uncertainties, especially due to the decreasing SNR as the shower energy decreases. To investigate the impact of shower energy on $X_{\max }$ uncertainties, we have varied the energy of the input events. Here we also folded an uncertainty of $20 \%$ in the energy of the input event, as expected from the first energy estimate uncertainties (see section 3.1). On the bottom left panel of Fig. 3 we show $\sigma_{X_{\max }}$ (solid lines) and the average error $<X_{\max }$ error $>$ (dashed lines) as a function of shower energy. To speed up the analysis, we did not include core position errors in this set and only applied the basic cuts. One can see that not only $\sigma_{X_{\max }}$ increases as we decrease the shower energy, but the bias $<X_{\max }$ error $>$ also increases. This shows that in order to perform quality reconstructions below $\sim 10^{16} \mathrm{eV}$ other approaches may be needed, such as hierarchical beam forming of subsets of antennas to increase SNR. Such an approach is under investigation. On the bottom right panel of Fig. 3 we show the final energy uncertainty $\sigma_{E}$ (solid lines) and the average error $\left\langle E_{\text {error }}\right\rangle$ (dashed lines) as a function of shower energy. Although the uncertainty in energy is more stable than that in $X_{\max }$, the

\footnotetext{
${ }^{1}$ For low zenith angles, a cut in the minimum number of antennas is almost equivalent to a cut on the maximum distance to the center of the array.

${ }^{2} \mathrm{~A}$ more refined spatial cut for larger zenith angles is in development. Preliminary results show that these cuts are able to significantly further reduce the uncertainty of more inclined showers down to $\sim 20 \mathrm{~g} / \mathrm{cm}^{2}$ at the expense of a reduced event rate.
} 

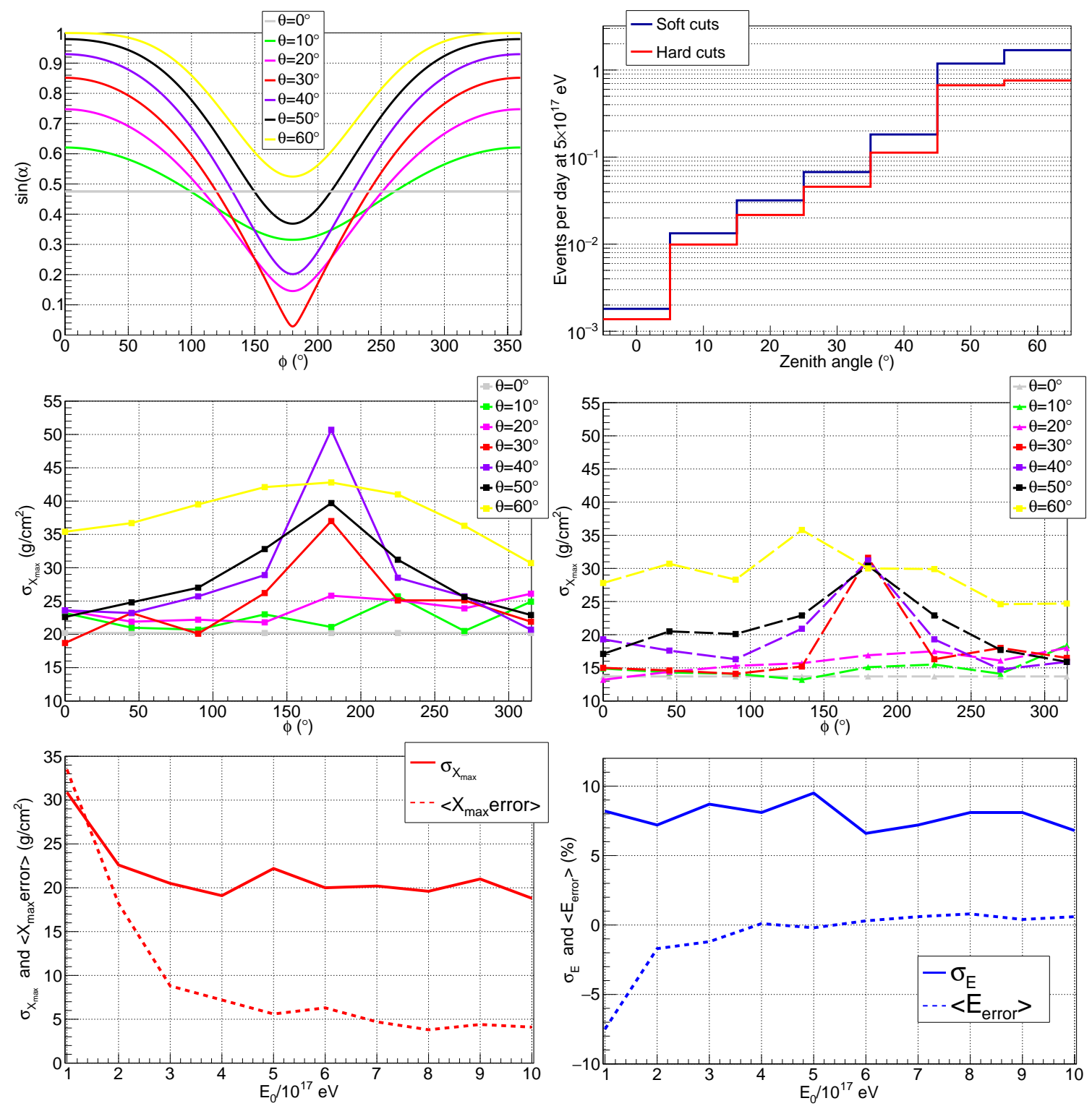

Figure 3: Top left: Expected scaling of the geomagnetic component of the shower $(\sin \alpha)$ as a function of the azimuth angle of the shower for several zenith angles. Top right: Expected event rate for $5 \times 10^{17} \mathrm{eV}$ high quality events using the normal soft cuts (blue) and harsher cuts (red). Middle left: $X_{\max }$ uncertainty as a function of azimuth angle for several zenith angles, using normal cuts. Middle right: Same as middle left, but using the harsher cuts. Azimuth angles are shown in the AIRES coordinate system, where $\phi=0^{\circ}$ is North and $\phi=90^{\circ}$ is West. Bottom left: $X_{\max }$ uncertainty (red solid line) and average $X_{\max }$ error (red dashed line) as a function of shower energy. A $20 \%$ energy uncertainty was folded into the input event, as expected by the first estimates shown in Section 3.1, and only the basic soft cuts were applied. Bottom right: Final energy uncertainty (blue solid line) and final average energy error (blue dashed line) as a function of shower energy. Here we also folded a $20 \%$ energy uncertainty into the input events and only applied the basic soft cuts. 
bias in the energy reconstruction also increases as shower energy decreases. Note that we assumed a perfect knowledge of the effective height of the antennas, disregarding any errors that would lead to a systematic energy error. Such effects will be studied in a future work.

\section{Conclusions and prospects}

We have estimated the expected performance of $X_{\max }$, energy and core position reconstructions using the OVRO-LWA-352 expansion. Our simulations show that using a set of simple cuts, $X_{\max }$ uncertainties lower than $20 \mathrm{~g} / \mathrm{cm}^{2}$ for the majority of arrival directions are possible. We have also shown that due to the larger antenna spacing away from the central core, the effective area for quality detection of more vertical showers is much smaller than that for larger zenith angles. We have also investigated the dependence of the $X_{\max }$ uncertainty with energy and arrival direction, the latter arising not only due to the $\sin \alpha$ scaling of the electric field, but also due to the asymmetric character of the array, creating the need for quality cuts that depend on arrival direction. The creation of more refined spatial cuts for inclined showers, based on large numbers of simulated events, is underway. We have also shown that uncertainties in the reconstructed energy, at least those that are intrinsic to the method and those due to galactic noise, are very small $(\lesssim 20 \%)$, as are the uncertainties in core position, which are lower than $\sim 17 \mathrm{~m}$ even for the most inclined events. At lower energies $\left(\lesssim 10^{17} \mathrm{eV}\right)$, we expect the $X_{\max }$ uncertainties to increase significantly if using the presented method. We are currently investigating alternative methods to determine the composition of lower energy events, such as increasing SNR by using hierarchical beam-forming on subsets of antennas and timing-based reconstructions of the radio front shape [9, 10]. We also intend to create parametrizations for the superposition model in [5] so that the creation of dedicated sets of simulations for each event is no longer needed. Finally, corrections to $X_{\max }$ measurements due to fluctuations in the refractive index of the atmosphere will be created in the future.

\section{acknowledgments}

W.R.C. thanks grants \#2015/15735-1 and \#2018/18876-3, São Paulo Research Foundation (FAPESP). KP is supported by a National Science Foundation Graduate Research Fellowship.

\section{References}

[1] R. Monroe et al., submitted for publication to Nucl. Instrum. Meth. A.

[2] F. Schröder et al., arXiv:1903.07713 [astro-ph.HE], 2019

[3] P. Abreu et al. (Auger collaboration), Astrophys. J., 760, 148, 2012

[4] J. Alvarez-Muñiz, W.R. Carvalho Jr., E. Zas, Astropart. Phys. 35, 325-341, 2012

[5] J. Alvarez-Muñiz, W. R. Carvalho, H. Schoorlemmer, E. Zas, Astropar. Phys 59, 29-38, 2014

[6] S. Buitink et al (LOFAR collaboration), Phys. Rev. D 90, 082003, 2014 and Nature 531, 70, 2016

[7] F. Schröder, Prog.Part.Nucl.Phys. 93, 1-68, 2017

[8] W. R. Carvalho Jr and J. Alvarez-Muñiz, Astropar. Phys. 109, 41-49, 2019

[9] A. Corstanje et al., Astropar. Phys. 61, 22-31, 2015

[10] W.D. Apel et al., JCAP09(2014)025A, 2014 